\title{
Subarachnoid haemorrhage and the electrocardiogram - a review
}

\author{
ANTHONY D. HARRIES \\ M.A., M.R.C.P., D.T.M. \& H. (Liverpool) \\ Department of Medicine and Gastroenterology, University Hospital of Wales, Heath Park, Cardiff
}

\begin{abstract}
Summary
Electrocardiographic abnormalities occur in approximately $50 \%$ of patients with subarachnoid haemorrhage. The changes can resemble those of myocardial damage, although in most cases post-mortem studies show no macroscopic evidence of injury. Failure to appreciate this can lead to misdiagnosis of organic cardiac disease, cause delay in surgical treatment or even lead to inappropriate therapy. This review deals with the changes that may occur in the electrocardiogram, discusses the explanations put forward, and examines how this may influence future trends in management.
\end{abstract}

The abnormalities in the electrocardiogram

The association of ECG abnormalities and subarachnoid haemorrhage has been known for at least 30 years (Byer, Ashman and Toth, 1947), and the changes documented in the literature are listed in Table 1. The incidence and type of change that can

TABLE 1. Possible ECG changes in subarachnoid haemorrhage

\begin{tabular}{ll}
\hline P Wave & $\begin{array}{l}\text { TALL and PEAKED (exceeding } 2.5 \mathrm{~mm} \\
\text { in lead II) }\end{array}$ \\
P - R Interval & $\begin{array}{l}\text { Prolonged or shortened } \\
\text { Pathological }\end{array}$ \\
Q Wave & Exceeding $35 \mathrm{~mm}$ \\
SV1 + RV5 & Elevated or depressed \\
ST Segment & Peaked : Flattened : Inverted \\
T Wave & Prolonged or shortened \\
Q - Tc Interval & TALL (exceeding $1 \mathrm{~mm}$ ) or Inverted \\
U Wave & \\
\hline
\end{tabular}

occur varies widely from one patient to another, and is influenced by the patient's previous cardiovascular status (Hunt, McCrae and Zapf, 1969). An abnormal $\mathrm{U}$ wave is one of the commonest changes to occur in subarachnoid haemorrhage, with an incidence of between $50-60 \%$ and, following this, changes of the ST segment, T wave and Q-Tc interval are found in approximately $50 \%$ of the patients (Eisalo, Peräsalo and Halonen, 1972). The other abnormalities are found less frequently, but in general it is fair to state that these figures are probably an underestimate of their true occurrence. Unless daily tracings are done, ECG abnormalities are likely to be overlooked 3 because they do not always occur at the outset of the if haemorrhage and may be delayed for one or 2 . weeks. The duration of change is also variable. In $\stackrel{\text { o }}{ }$ many patients who survive, the abnormal ECG is reverts to normal within 2 weeks, although changes $\stackrel{\circ}{\perp}$ can persist up to 6 weeks after the initial cerebro- 음 vascular insult (Shuster, 1960).

Disorders of cardiac rhythm also occur. These $\bar{z}$ include sinus bradycardia and tachycardia, sinus arrhythmia unrelated to phase of respiration, and $\overrightarrow{0}$ supraventricular or ventricular arrhythmias. Theg $\infty$ can sometimes in their own right be life-threatening

The genesis of the ECG changes

Electrolyte disturbances, in particular a low serum $\bar{O}$ potassium, have been postulated to cause the ECG $\frac{\mathrm{D}}{\square}$ abnormalities (Hersch, 1964). Recent work, how- $\varrho$ ever, throws doubt on this explanation, although $\overrightarrow{\vec{O}}$ Cruickshank, Neil-Dwyer and Stott (1974b) found 3 that there was a correlation with low levels of total body exchangeable potassium.

Cropp and Manning (1960) attributed the ECG changes to irritation of area 13 on the orbital surfaces of the frontal lobes. The vagus nerve is thought $\frac{5}{3}$ to have cortical representation in this area, and operative manipulation of its blood supply will pro- $\frac{\text { 의 }}{3}$ duce abnormal electrocardiogram and associated ventricular arrhythmias. This theory could explain $\frac{}{2}$ changes occurring with anterior cerebral artery $\rightarrow$ aneurysms, but not with those in the posterior fossa. Some of the ECG changes, however, do compare N with those seen in experimental vagal stimulation, and atropine will certainly reverse some of the abnormalities (Shuster, 1960). Intense sympathetic tone $\omega$ was put forward as the cause of ischaemic changes (Burch, De Pasquale and Malaret, 1960), and later $\stackrel{C}{C}$ Melville et al. (1963) were able to show that both the sympathetic and parasympathetic nervous supply were responsible in part for the abnormal ECG ${ }^{0}$ changes observed in the experimental situation with $\underset{\mathbb{D}}{\stackrel{D}{(19}}$ stimulation of the cat hypothalamus. 
Attention was refocused on the heart when Koskelo, Punsar and Sipila (1964) described subendocardial haemorrhage in association with subarachnoid haemorrhage, and Hoff brand and Morgan (1965) later showed that cardiac function could also be significantly impaired. In a significant number of patients with subarachnoid haemorrhage, there are increased levels of creatinine phosphokinase-muscle enzyme (Dubo et al., 1967), and it was suggested that this originated from cardiac damage (Connor, 1967). Careful post-mortem studies on macroscopically normal hearts revealed histological lesions in the form of focal myocytolysis (Connor, 1968), and a recent study by Doshi and Neil-Dwyer (1980) showed that similar lesions could be found in $80 \%$ of the patients coming to post-mortem. In this study, ECG abnormalities occurred in $70 \%$ of the patients with myocardial lesions, and the incidence could have been higher if daily ECGs had been routine.

The other histological finding of major importance in resolving the problem was hypothalamic damage, first described 17 years ago (Crompton, 1963). Recent studies have shown that hypothalamic lesions in the form of perivascular haemorrhage, oedema and even frank infarction occur in the majority of such patients coming to post-mortem (Doshi and Neil-Dwyer, 1980). Recently, attempts have been made to correlate the hypothalamic and myocardial lesions (Doshi and.Neil-Dwyer, 1977), and the more recent study by Doshi and Neil-Dwyer (1980) shows there to be a strong association.

Cerebrovascular spasm is a well recognized accompaniment of subarachnoid haemorrhage. Spasm affecting the small hypothalamic blood vessels will lead to lesions in that area, and this in turn will cause an abnormal hypothalamic response, with increased catecholamine production via the sympathetic system (Cruickshank, Neil-Dwyer and Brice, 1974a), and increased circulating steroid levels via the pituitary-adrenal axis (Jenkins et al., 1969). Catecholamines can produce ECG abnormalities in subjects with no clinically apparent heart disease (Lepeschkin et al., 1960) and can produce cardiac lesions identical with those occurring in subarachnoid haemorrhage (Reichenbach and Benditt, 1970). Corticosteroids are thought to play a role too in potentiating the cardiotoxic effects of catecholamines, as they decrease total body exchangeable potassium, which in turn probably depletes myocardial intracellular potassium, thereby rendering the cell prone to injury (Selye, 1970).

Further evidence that the autonomic nervous system is responsible for subendocardial necrosis and ECG abnormalities comes from animal experiments on simulated subarachnoid haemorrhage where both abnormalities can be prevented by surgical (C2 cordotomy) or by medical (propranolol) interven- tion (Greenhoot and Reichenbach, 1969). In patients also, propranolol will reverse many of the ECG changes (Cruickshank, Neil-Dwyer and Lane, 1975), and atropine will abolish changes such as T-wave peaking which are thought to result from increased vagal tone. A recent study, again by Neil-Dwyer et al. (1978), showed that propranolol was probably the agent instrumental in preventing myocardial damage and ECG changes.

There is therefore strong evidence to support the theory that subarachnoid haemorrhage via the hypothalamus causes an abnormal activation of the autonomic nervous system and that this is responsible for the electrocardiographic abnormalities.

\section{The ECG in prognosis}

It was originally thought that the occurrence and persistence of ECG changes was unrelated to the course of the illness (Shuster, 1960; Ananthachari and Anto, 1967), although it had been recognized that atrial fibrillation was associated with a bad prognosis. Cruickshank et al. (1974a), however, in a study involving a larger number of patients, were able to show that certain ECG changes could serve as prognostic indicators. Pathological $Q$ waves and raised ST segments herald a uniformly bad prognosis. Various combinations of peaked $\mathbf{P}$ wave, short $\mathbf{P}-\mathbf{R}$ interval, long $\mathrm{Q}-\mathrm{Tc}$ interval, tall $\mathrm{U}$ wave and peaked $\mathrm{T}$ wave appear to be associated with a poor outlook irrespective of the level of consciousness, the degree of angiographically demonstrated spasm or the type of treatment. These changes, with the exception of peaked $T$ waves, are found with increased catecholamine production, and therefore presumably reflect the degree of autonomic nervous system derangement which in turn is related to the degree of spasm and the severity of the initial haemorrhage.

\section{Relationship to medical management}

The abnormal cardiovascular response in subarachnoid haemorrhage may in some cases contribute to increased morbidity and mortality. Studies so far have failed to show any influence on mortality of combined $\alpha$ - or $\beta$-blockage (Neil-Dwyer et al., 1978 ), although $\beta$-blockage is cardioprotective. The reason is that potentially fatal secondary events such as renewed bleeding are not critically influenced by these medical agents, but nevertheless cardioprotective treatment may well save the lives of a small number of patients whose intracranial lesions are not lethal in their own right. Similarly, in other stress areas such as head injuries, where myocardial necrotic lesions and unstable cardiac rhythms can occur, $\beta$-blockade may have a useful role.

Cardiac transplantation is now becoming an accepted mode of therapy for end-stage cardiac 
disease, and the source of donors is another factor to be considered. The potential cardiac donors are often patients with severe head injuries or with severe cerebro-vascular accidents, and consideration should be given to prophylactic $\beta$-blockade to enable the recipient to have, as near as possible, a heart protected from the deleterious effects of an abnormally charged autonomic nervous system.

\section{Acknowledgment}

The author would like to thank Dr J. R. Harries for his help in preparing the manuscript.

\section{References}

Ananthachar!, M.D. \& Anto, C.D. (1967) A study of ECG changes in 20 cases of subarachnoid haemorrhage. Indian Heart Journal, 19, 105.

Burch, G.E., De Pasquale, N. \& Malaret, G. (1960) Selected problems in electrocardiography. Annals of Internal Medicine, 52, 587.

Byer, E., Ashman, R. \& Toth, L.A. (1947) Electrocardiograms with large upright $\mathrm{T}$ waves and long $\mathrm{Q}-\mathrm{T}$ intervals. American Heart Journal, 33, 796.

ConNoR, R.C.R. (1967) Creatinine kinase in intracranial lesions. Lancet, ii, 991 .

ConNOR, R.C.R. (1968) Heart damage associated with intracranial lesions. British Medical Journal, 3, 29.

Crompton, M.R. (1963) Hypothalamic lesions following the rupture of cerebral berry aneurysms. Brain, 86, 301.

Cropp, G.J. \& ManNing, G.W. (1960) Electrocardiographic changes simulating myocardial ischemia and infarction associated with spontaneous intracranial hemorrhage. Circulation, 22, 25.

Cruickshank, J.M., Neil-Dwyfr, G. \& Brice, J. (1974a) Electrocardiographic changes and their prognostic significance in subarachnoid haemorrhage. Journal of Neurology, Neurosurgery and Psychiatry, 37, 755.

Cruickshank, J.M., Neil-Dwyer, G. \& Lane, J. (1975) The effect of oral propranolol upon the ECG changes occurring in subarachnoid haemorrhage. Cardiovascular Research, 9, 236.

Cruickshank, J.M., Neil-Dwyer, G. \& Stott, A.W. (1974b) Possible role of catecholamines, corticosteroids and potassium in the production of electrocardiographic abnormalities associated with subarachnoid haemorrhage. British Heart Journal, 36, 697.

Doshi, R. \& NeIL-DWYer, G. (1977) Hypothalamic and myocardial lesions after subarachnoid haemorrhage. Journal of Neurology, Neurosurgery and Psychiatry, 40, 821.
Doshi, R. \& NeIL-Dwyer, G. (1980) A clinico-pathological study of patients following a subarachnoid hemorrhage.
Journal of Neurosurgery, 52, 295 .

Dubo, G., Park, D.C., Pennington, R.J.T., Kalbag, R.M.C. \& WALTON, J.N. (1967) Serum creatine kinase in cases of stroke, head injury and meningitis. Lancet, ii, 743.

Eisalo, A., Peräsalo, J. \& Halonen, P.I. (1972) Electrocardiographic abnormalities and some laboratory findings in patients with subarachnoid haemorrhage. British Heart Journal, 34, 217.

Greenhoot, J.H. \& ReIChenbach, D.D. (1969) Cardiacid injury and subarachnoid hemorrhage. A clinical, pathological and psychological correlation. Journal of Neuro-心 surgery, 30, 521.

HersCH, C. (1964) Electrocardiographic changes in sub-. arachnoid haemorrhage, meningitis and intracranial $\overrightarrow{\vec{\omega}}$ space-occupying lesions. British Heart Journal, 26, 735.

HoffBrand, B.I. \& Morgan, B.D.G. (1965) Functiona! sig $=\frac{\Omega}{8}$ nificance of electrocardiographic changes associated w:tho subarachnoid haemorrhage. Lancet, i, 844.

Hunt, D., McCrae, C. \& Zapf, P. (1969) Electrocardio-i graphic and serum enzyme changes in subarachnoid. hemorrhage. American Heart Journal, 77, 479.

Jenkins, J.G., Buckell, M., Carter, A.B. \& Westlake, S.ন (1969) Hypothalamic-pituitary-adrenal function afterĩ subarachnoid haemorrhage. British Medical Journal, 4,® 707.

Koskelo, P., Punsar, S. \& Sipila, W. (1964) Subendocardial haemorrhage and ECG changes in intracranial bleeding. British Medical Journal, 1, 1479.

Lepeschinin, E., Marchet, H., Schroeder, G., Wagner, R., de Paula e Silva, P. \& RaAb, W. (1960) Effect $8 f_{0}$ epinephrine and noradrenaline on the electrocardiogran $\infty$ of 100 normal subjects. American Journal of Cardiology, 594.

Melville, K.I., Blum, B., Shister, H.E. \& Silver, M.尹. (1963) Cardiac ischemic changes and arrythmias induced by hypothalamic stimulation. American Journal of Cardio$\log y, 12,781$.

Neil-Dwyer, G., Walter, P., Cruickshank, J.M., Doshi, B. \& O'Gorman, P. (1978) Effect of propranolol and $=$ phentolamine on myocardial necrosis after subarachnoid윽 haemorrhage. British Medical Journal, 2, 990.

ReICHENBACH, D.D. \& BenditT, E.P. (1970) Catecholamines and cardiomyopathy: the pathogenesis and potentialo. importance of myofibrillar degeneration. Human Pathology, $1,125$.

SELYE, H. (1970) The evolution of the stress concept. American Journal of Cardiology, 26, 289.

SHUSTER, S. (1960) The electrocardiogram in subarachnoid haemorrhage. British Heart Journal, 22, 316. 